\title{
Zur Diagnose und Therapie tuberkulöser Hautaffektionen.
}

Von Dr. Franz Nagelsehmidt in Berlin.

Die Erkenntnis bricht sich mehr und mehr Bahn, dafl seit der systematischen Einführung der Finsenbehandlung die Ausbreitung des Lupus einen deutlich erkennbaren Rückschritt gemacht hat. Es ist nicht zu bezweifeln, daB sowohl für die kleinen, frischen Lupusfälle, als auch für ausgedehnte, ältere, die Lichtbehandlung nach $F$ ins en vermöge ihrer kosmetischen Resultate die Behandlung katexochen ist. Trotzdem würde man den Vorwurf der Einseitigkeit auf sich laden, wenn man nun wahllos jeden Lupusfall ausschlieflich mit Licht behandeln wollte. Es ist hier nicht der Ort, auf die spezielle Indikation der Behandlung der verschiedenen Lupus- und Hauttuberkulosearten einzingehen. Ich möchte nur hervorheben, daß, wenn auch die Lichtbehandlung die Hauptbehandlungsmethode ist und bleiben wird, wir doch in einzelnen Fällen gezwungen sein können, andere Behandlungsmethoden zul Vorbereitung oder zur Nachbehandlung anzuwenden, und jede Bereicherung unseres Arsenals im Kampfe mit der Hauttuberkulose ist zu begrüßen, besonders, wenn es sich um eine leicht ausführbare und bequeme Methodik handelt.

Bei der Lichtherapie sind es vor allem zwei Punkte, die dem Arzte wohl in jedem der Abheilung nahen Falle Schwieligkeiten bereiten. Erstens die Diagnose: Ist der Lupus wirklich heil, oder sitzt doch hier und da noch ein versprengter Keim, der durch die noch nicht abgelaufene Behandlungsreaktion verdeckt wird und erst später in die Erscheinung tritt? Der zweite Punkt ist das Bestehenbleiben vereinzelter Lupusknötchen, besonders, wenn sie in derbem Narbengewebe eingebettet sind und so selbst intensiven und häufig wiederholten Bestrahlungen Widerstand leisten. Es ist eine alltägliche Beobachtung, daß der Lichtbehandlung im ganzen gut zugängliche Stellen doch hier und da ein oder mehrere Knötchen aufweisen, die mitunter eine 20- und 30malige Bestrahlung nötig machen, um scheinbar abzuheilen und dann doch nach einigen Monaten zil rezidivieren.

lch habe vor einigen Jahren die Methode der lokalen Blutuntersuchungen ausgearbeitet und dariber in der Berliner Medizinischen Gesellschaft referiert. Es hat sich dabei in zahireichell Fällen gezeigt, daß in tuberkulösen Hautherden bei flachem Einstich direkt in den Hord dor zuerst austretende lilutstropfen eine starko Vormoblung der Lymphocyten gegeniiber den Zahlen des Allgeneinblutes aufweist, und es ist mir 
wiederholt in diagnostisch schwierigen Fällen gelungen, eine tuberkulöse Affektion von einer tertiär syphilitischen hierdurch zu unterscheiden. Aber die Verhältnisse des lokalen Blutstropfens sind viel zu sehr von zufälligen mechanischen Einflüssen (Palpation vor der Untersuchung etc.) abhängig, als daß nan bei negativem Ausfall der Untersuchung Schlüsse ziehen könnte. Auch die Methode des einfachen Glasdrucks, sowie die Liebreichsche mittels des Strahlenkegels sind so sehr dem subjektiven Ermessen und daher größten Irrtümern unterworien, daß trotz größter persönlicher Erfahrung die sichere Feststellung unmöglich ist, ob definitive Heilung eingetreten ist oder noch Reste vorhanden sind. Ich habe mich in solchen Fällen häufig der üblichen diagnostischen subkutanen Injektion von Alttuberkulin bedient, muß aber sagen, dab eindeutige Re. sultate håufig in solchen Fällen erzielt wurden, wo schon klinisch die Diagnose nicht zweifelhaft war; aber in vielen Fällen versagt die lokale Reaktion trotz Auftretens einer Allgemeinreaktion und auch ohne diese. Mitunter ist es sehr schwer, minimale Farbendifferenzen von einem Tage zum andern aus der Erinnerung zu vergleichen, und häufig verhindert das $\mathrm{Be}$ stehen eines tuberkulösen Lungen- oder Knochenherdes etc., so grobe Dosen anzuwenden, wie sie zur Hervorrufung der Hautreaktion in tuberkulös erkrankten Hautgebieten mitunter erforderlich wären.

Diese Tuberkelinjektionen werden auch von manchen Aerzten systematisch zur ambulanten Behandlung des Lupus angewandt, indem minimale Tuberkulinmengen chronisch injiziert werden; immerhin ist man bei dieser Methode gezwungen, um einen mitunter nur kleinen Hautherd zu behandeln, den ganzen Organismus der Tuberkulinbehandlung zu unterwerfen, und es besteht die Gefahr, dab gerade bei ambulanter Behandlung die allgemeine Reaktion, wenn sie doch einmal eintritt, sich als schädlich geltend macht.

Es war daher mein Bestreben, die an sich als zuverlässig erprobte Reaktion tuberkulösen Gewebes auf Tuberkulin oder' tuberkulinähnliche Präparate möglichst lokal unter Schonung des Gesamtorganismus verlaufen zu lassen, und ich begann meine Versuche der lokalen Tuberkulinimpfungen zunächst lediglich aus diagnostischen Gründen. Die Methodik ist einfach und gegeben. Es genügt die Herstellung einer kleinen Exkoriation mittels irgend eines Instrumentes, Pinzette, Lanzette oder auch des jüngst angegebenen v. Pirquetschen Drehspatels, sowie die Beschickung des kleinen, oberflächlichen Defektes mit Tuberkulin. Ich habe mich in meinen Versuchen ausschlie日lich des in seinen Wirkungen genügend bekannten und erprobten $\mathrm{Kochschen} \mathrm{Alttuberkulins} \mathrm{be-}$ dient. Von einer Dosierung habe ich bald Abstand genommen.

Es ist ganz gleichgültig, ob man einen Tropfen genuinen oder 10-, 100- oder 1000-fach verdünnten Tuberkulins anwendet. Die Menge, die notwendig ist, um ohne Verdünnung durch die Blutbahn, an Ort und Stelle mit den Toxinen, resp. Gewebsbestandteilen, in Reaktion zu treten und zu einer sichtbaren Reaktion zu führen, ist so verschwindend klein, dah ein Tropfen der 1000-fachen Verdünnung schon ein Vielfaches der notwendigen Quantität darstellt. Da nun die Reaktionsfähigkeit der Haut der verschiedenen Menschen gegenüber dem Tuberkulin, wie dies auch von v. Pirquet und seinen Nacharbeitern konstatiert worden ist, eine außerordentlich verschiedene sein kann, habe ich stets außer dem tuberkulösen, resp. tuberkuloseverdächtigen Herd in der Entfernung von $1-2 \mathrm{~cm}$ auch die normal erscheinende Haut geimpft. Der Mangel der Pirquetschen Hautimpfmethode als Diagnosticum einer innerlich lokalisierten Tuberkulose liegt in dem Auftreten der Reaktion fast bei jedem Erwachsenen. Dieser Mangel haftet der Lokalimpfung im Hautherde nicht an, insofern als die Reaktion bei normaler Haut auch beim tuberkulösen Erwachsenen wesentlich anders als im tuberkulösen Herde verläuft. Während bei der gesunden Haut sich eine Papel nach einigen Stunden bildet, die sich mitunter schon nach wenigen Tagen mit einer kleinen Kruste bedeckt, und die Reaktion einen durchaus harmlosen Charakter zeigt, kommt es in tuberkulösen Herden häufig, ja fast mit Regelnı̈̈figkeit zur Geschw ürs bild ung; die Tiefe, die Ausdehnung des Substanzverlustes, sowie die Dauer der Eiterung kann in weiten Grenzen wechseln und ist von Faktoren ab- hängig, bei denen ich noch keine Gesetzmäßigkeit habe konstatieren können. Offenbar spielt hierbei die Virulenz des Prozesses einerseits und die lokale Reaktionsfähigkeit des Organismus anderseits eine bedeutende Rolle. Um den Verlauf zu zeigen, möchte ich einige Beispiele kurz anführen.

Fall 1. 14 jähriger Junge; isolierter, elliptischer, $6 \mathrm{~cm}$ langer, $3 \mathrm{~cm}$ breiter Herd von Tuberculosis cutis verrucosa in der Nähe des rechten Handgelenks. Am Tage nach der Impfung ist die rechte Cubitaldrüse geschwollen, $n$ icht $\mathrm{schmerzhaft,} \mathrm{starke} \mathrm{Reaktion} \mathrm{des}$ ganzen Herdes bis $1 \mathrm{~cm}$ darüber hinaus. Am nächsten Tage ist der Herd noch rot gefärbt, ödematös und in der Umgebung der drei Impfpunkte mit kleinen Bläschen bedeckt.

Die Impfpunkte sind exkoriiert, mit bräunlichen Krusten bedeckt; Berührungsempfindlichkeit besteht nicht. Die Reaktion läuft innerhalb von sieben Tagen fast ab; noch nach zehn Tagen sind Reste (Schuppung) deutlich erkennbar.

Fall 2. Neunjähriges Mädchen; wenig großer Herd auf dem linken Oberarm; am Tage nach der Impfung ist der Lupusherd und seine direkte Umgebung im Durchmesser von etwa $6 \mathrm{~cm}$ ödematös durchtränkt, stellenweise Blasenbildung. Nach drei Tagen ist der Lupusherd noch von einer kreisförmigen Blase umgeben, die am nächsten Tage zu einer Borke eintrocknet. Noch nach elf Tagen ist der Herd leicht geschwollen und hyperämisch.

Fall 3. Erwachsener; Lupus am Halse. Fünf Tage nach der Impfung noch kleines, eiterndes Ulcus in Zentrum. Nach acht Tagen Reaktion deutlich, nach elf abgelaufen.

In allen Fällen war die Reaktion, die zur Kontrolle an gesunder Hautstelle hervorgerufen wurde, wesentlich milder in ihrem Verlauf und längst abgeheilt, in manchen Fällen sogar nicht mehr erkennbar, während die Reaktion im tuberkulösen Gewebe noch in voller Entwicklung stand. Bemerkenswert nun und übereinstimmend mit unsern sonstigen Kenntnissen von der Impfung ist, daß bei wiederholter Inokulation desselben Herdes der Verlauf sich zu ändern pflegt. Während die erstmalige Reaktion mitunter erst nach zwei bis drei Tagen ihr Maximum erreicht und acht bis zehn Tage oder länger zum Ablauf gebrauchen kann, drängt sich bei späteren Impfungen der ganze Erscheinungsverlauf zusammen. Die Reaktion kann schon nach Stunden einsetzen, erreicht schon am nächsten Tage ihre Akme und läuft schon in wenigen Tagen ab.

Z. B. Erste lmpfung am 20. Juli. Beginn der Reaktion am nächsten Tage. Am 25. Juli. Impfung an einer andern Stelle. Die alte Reaktion ist noch nicht abgelaufen. Zwei Tage später sind die ersten Impfstellen noch in starker Reaktion, während die zweiten viel weniger reagieren. Am 31. Juli sind sümtliche Reaktionen abgelaufen. Am 12. August nochmalige Impfung. Am 16. August, also schon nach vier Tagen, Reaktion abgelaufen. Am 4. September zum vierten Male geimpft. Am 7. September Reaktion fast abgelaufen bis auf kleine Exkoriationen, die als nicht mehr spezifische Reste aufzufassen sind.

Um die Spezifizität der Reaktion festzustellen, habe ich auch andere Hauteffloreszenzen geimpft, z. B. syphilitische. Auch diese haben dio Hautreaktion, wie sie bei Erwachsenen normal ist, gezeigt. Indessen ist in keinem Fall das Auftreten nennenswerter Ulzerationell beobachtet worden. Bei minimalen Herden, resp. Resten von solchen kann auch in Fällen, wo unbedingt Lupus vorher bestanden hat, der Grad der Reaktion ein sehr geringer sein; immerhin wird man aber von einem positiven Ausfall der Reaktion dann sprechen können, wenn der Reaktionsgrad zwischen Impfistellen und normaler Hautstelle deutlich verschieden ist. Ich schätze diesbezüglich den diagnostischen Wert der Reaktion sehr hoch ein. Ob andere Hauteffloreszenzen unter Umständen in ähnlicher Weise reagieren können, wird Sache der weiteren Untersuchung sein.

Bei den oben erwähnten diagnostischen Impfungen habo ich nun die Beobachtung gemacht, daB in einzelnen Fällen mit dem Ablauf der Reaktion auch eine klinische Heilung oder Besserung der Effloreszenzen eingetreten ist, und ich habe nunmehr die Versuche unter dem Gesichtspunkt der therapeutischen Beeinflussung fortgeführt. Ich habe zu meiner Freude konstatieren können, daß eine Anzahl zirkumskripter Lupusknötchen und lupöser Infiltrate, die nur mit lokaler Impfung behandelt wurden, zurzeit klinisch abgeheilt sind. $O b$ es sich an diesen Stellen um eine definitive Heilung handelt, kann natürlich jetzt nicht entschieden werden. Denn bekanntlich wird auch für die Beurteilung der Heilung eines 
Lupus, z. B. durch Finsenbehandlung, ein zweijähriges Freibleiben von Rezidiven verlangt.

fall 4. Knabe von zwolf Jahren. Trotz wiederholter Finsenbehandlung treten bei einem talergroßen Herde der linken Wange stets wieder Rezidive kurze Zeit nach Aussetzen der Behandlung auf. Am Tage nach der Impfung beginnende Lokalreaktion, die nach fïnf Tagen abzuheilen beginnt. Nach zweieinhalbmonatiger Beobachtungszeit frei von Rezidiven.

Wenn somit die Möglichkeit besteht, inittels der lokalen Tuberkulinimpfung einzelne tuberkulöse Effloreszenzen zur Einschmelzung und vorläufigen - - vielleicht auch definitiven - Heilung zu führen, so haften der Methode doch große Nachteile an. Zunächst leidet sie ebenfalls an dem Kardinalfehler der Finsenbehandlung, d. h. das therapoutische Wirkungsbereich erstreckt sich nur auf wenige Millimeter im Umkreis der Impfung, wenngleich der Umfang der Reaktion mitunter $5 \mathrm{~cm}$ und mehr an Ausdehnung aufweist. Ein woiterer Nachteil liegt darin, daB wir durch die Impfung oder bei der Impfung den kosmetischen Effekt in keiner Weise beherrschen. Wie anfänglich mitgeteilt, findet auch bei starker Verdünnung mitunter eine intensive Reaktion statt, und wir können einstweilen die Tiefe und die Ausdehnung des Substanzverlustes nicht beeinflussen. Da nun bei positivem Ausfall der Reaktion in jedem Fall eine Eiterung und somit ein Substanzverlust stattfindet, so kommt es stets zur Bildung einer Narbe, und der kosmetische Effekt kann weit hinter dem der Finsenbehandlung zurückstehen. Anderseits wird man doch in manchen Fällen zu dieser Behandlung greifen können, besonders wenn es sich um vereinzelte, gegen Finsenbehandlung refraktäre Herde handelt, die bereits in Narbengewebe eingelagert sind, wobei man dem Patienten langweilige und kostspielige Lichtsitzungen durch einen einmaligen oder mehrere Male zu wiederholenden, kurzen Eingriff erspart. Vor dem chirurgischen und kaustischen Eingriff hat die Behandlung der lokalen Impfung den Vorteil, daß sie fast schmerzlos ist und daf sie elektiv das Tuberkulöse zerstört, ohne dabei zu einer Verschleppung Gelegenheit zu geben.

Ein fernerer Nachteil liegt in dem Umstand, daß trotz der minimalen Dosen, die mit der Wundfläche in Kontakt kommen, mehr oder weniger häufig Allgemeinreaktionen auftreten können. Z. B. sah ich bei einer Kranken mit ausgedehntem Lupus des Gesichts und des Halses sowie der Schleimhäute Fieber bis zu $38,1^{\circ}$ vier Tage lang auftreten und deutliche Reaktion der gesamten Lupusfläche, die mit Schmerzen und allgemeinem Krankheitsgefühl einherging. In einem andern Falle setzte ebenfalls eine deutliche Allgemeinreaktion mit starker Mattigkeit ein, ohne daß aber die Lupuspartien, die von der Impfstelle mehr als etwa $5 \mathrm{~cm}$ entfernt waren, eine erkennbare Reaktion aufwiesen. Um diese allgemeine Reaktion zu vermeiden, und da ich erkannte, daß minimalste Quantitäten zur Erzielung der lokalen Reaktion ausreichten, habe ich die Methodik in der Weise modifiziert, daß ich zwei oder drei Tropfen Tuberkulin an ebenso vielen Stellen deponierte, innerhalb. dieser Tropfen durch Schaben die Exkoriation erzeugte und nach 15 oder 20 Sekunden mittels eines trockenen Wattebausches, ohne die geimpften Stellen zu berühren, die Tropfen absaugte. Es blieb dann auf der Exkoriation genug von dem Impfstoff zurück, um die Reaktion auszulösen und die überflüssige Resorption, die zur Allgemeinreaktion führt, größtenteils zu vermeiden. Bei wiederholten Impfungen, bei denen, wie oben mitgeteilt, die Reaktion sich stark abschwächt, gelingt es im Gegensatz hierzn mitunter nicht, die nötige Tuberkulinmenge durch die Exkoriation resorbieren zu lassen. In diesem Falle spritze ich mit einer feinen Nadel einen kleinen Tropfen Tuberkulin direkt in das tuberkulöse Gewebe ein. Man kann auch sonst die Applikationsweise versehiedentlich modifizieren; z. B. kann man nach erfolgter Gewöhnung größere Flächen skarifizieren und dann die Impfung vornehmen.

Ich empfehle die lokale Impfmethode zur Nachprüfung und glaube, dab sie in geeigneten Fällen, zumal da, wo Lichtbehandlung - etwa auf dem Lande - nicht erreichbar ist, besonders auf vereinzelten Herden, therapeutische Erfolge erzielen kann. Für die Differentialdiagnose zwischen tuberkulösen und anderen Hautaffektionen, sowie für die Feststellung tuberkulöser Reste nach erfolgter anderweitiger Behandlung wird die Methode gelegentlich von Nutzen sein.

Ich lasse noch den mikroskopischen Befund eines Impfherdes auf lupösem Boden 48 Stunden nach der Impfung folgen.

Mikroskopischer Befund: Lupusherd, 48 Stunden nach der Impfung exzidiert. Man sieht intensive Fyperämie des ganzen Hautstückes bis zu mäßiger Tiefe. Capillaren stark dilatiert, strotzend gefüllt; am stärksten in unmittelbarer Umgebung des Lupusherdes. Daselbst Austritt von Ėrythrocyten, die, zum Teil zu Pigment zerfallen, frei im Gewebe liegen. Oedematorse Durchtränkung; intensive Zellinfiltration in den oberflachlichen Schichten, besonders in der Umgebung der Herde, aber auch diffus. Man sieht alle bei Entzündungen auftretenden Zellformen, überwiegend Granulationszellen, aber auch poly- und mononukleäre Leukocyten. Riesenzellen in guter A usbildung vorhanden. Es scheint, daß stellenweise eine Verkäsung durch eingewanderte Zellen verdeckt wird. Elastische Fasern, außer im Lupusknoten normal. Zwischen den Zellen findet sich ein fibrinartiges Filament. 